\title{
Report on Adaptive Force, a specific neuromuscular function
}

\author{
Marko Hoff, Laura Schaefer, Nancy Heinke, Frank Bittmann \\ Section Regulative Physiology and Prevention, Department of Sport and Health Sciences, \\ University of Potsdam, Germany
}

\begin{abstract}
In real life motions, as well as in sports, the adaptation of the neuromuscular systems to externally applied forces plays an important role. The term Adaptive Force (AF) shall characterize the ability of the nerve-muscle-system to adapt to impacting external forces during isometric and eccentric muscle action. The focus in this paper is on the concept of this neuromuscular action, which is not yet described in this way. A measuring system was constructed and evaluated for this specific neuromuscular function, but only the main information of the evaluation of the measuring system and the preliminary reference values are mentioned here, while an article with detailed description will be published separately. This paper concentrates on the three following points: 1) What is the peculiarity of this neuromuscular function, introduced as AF? 2) Is the measuring system able to capture its specific characteristics and which phases of measurement occur? 3) It seems reasonable to discuss if AF can be distinguished and classified among the known force concepts. The article describes the measuring system and how it is able to capture special features of real life motions like submaximal intensities and the subjects' option to react adequately on external varying forces. Furthermore, within one measurement the system records three different force qualities: the isometric submaximal Adaptive Force (AFiso), the maximal isometric Adaptive Force $\left(\mathrm{AFiso}_{\max }\right)$ and the maximal eccentric Adaptive Force $\left(\mathrm{AFecc}_{\max }\right)$. Each of these phases provide different and unique information on the nerve-muscle-system that are discussed in detail. Important, in terms of the Adaptive Force, seems to be the combination of conditional and coordinative abilities.

This project was funded by the Federal Ministry of Economy and Technology (Project ZIM KF2262301FO9).

Key Words: Adaptive Force, isometric-eccentric force, muscle action, pneumatic force measuring system
\end{abstract}

Eur J Transl Myol - Basic Appl Myol 2015; 25 (3): 183-189

Isometric muscle action means tension and force development without length variation of the muscle. Usually, the isometric force is detected by pushing with maximum force against an immovable restistance, i.e. a hand dynamometer. Thereby, the nerve muscle system acts maximally against the stable abutment. In real life, the isometric mode usually takes place at submaximal intensities and occasionally at maximal tension. Thus, while holding a weight the neuromuscular system must react appropriate to a consistent external force. It should apply exactly as much force as the holding weight requires for adapting to this load. This sensorimotor performance of adaptation is even more demanded if the external power requirements are changing temporally. The adaptive behavior of the nerve-muscle-system to variable external forces is the topic of this article. It is first determined under isometric conditions. But if the load exceeds the individual isometric force maximum the system merge into eccentric muscle action. This also requires adaptive behavior of the neuromuscular system and should be taken into account, too.

An example shall better illustrate the here considered neuromuscular proper functioning.

Imagine a person holding a big water canister while it is being filled. Thereby to hold this position, the nervemuscle-systems has to permanently adapt to the varying force requirements (due to the increasing load of the container), and thus to save the isometric condition. If the load increases above the maximum of the subjects' isometric force, the muscle passes over into yielding work. Also during yielding the system has to adapt further to the increasing load. This is why in the eccentric mode the muscle is able to reach about 


\section{Adaptive Force, a specific neuromuscular function}

Eur J Transl Myol - Basic Appl Myol 2015; 25 (3): 183-189

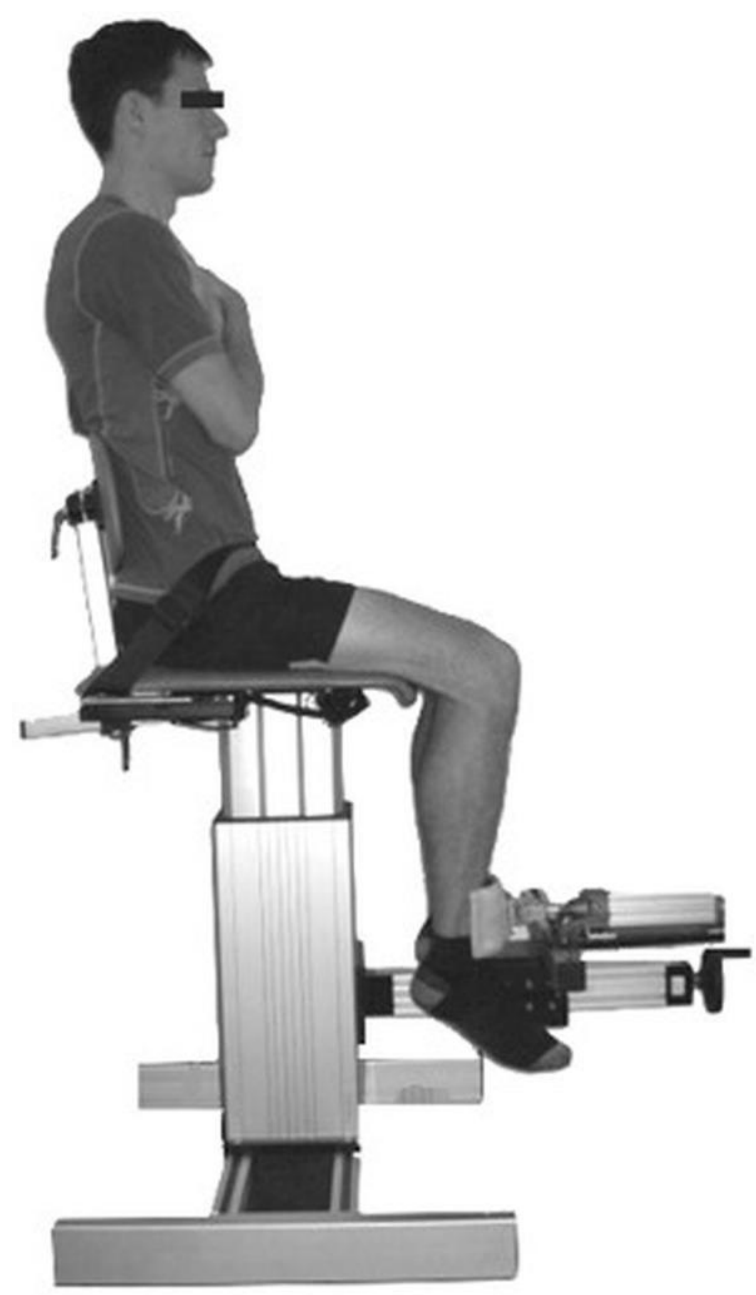

Fig 1. Measuring system "SeBit knee" for the detection of Adaptive Force $(A F)$ of the quadriceps femoris muscle.

$25-40 \%$ more maximal force than in the isometric mode. ${ }^{1}$ Only after exceeding the maximal eccentric force the resistance brakes down.

To this neuromuscular capacity of adaptation during isometric and eccentric functioning, respectively, we assigned the term „Adaptive Force“ (AF). Therefore, AF means the specific behavior of adaptation of the nerve-muscle-system to external forces. Whereby in submaximal area static conditions should be maintained (isometric: AFiso) and in supramaximal ranges the give in should be as slow as possible (eccentric: AFecc), respectively.

- On the one hand, AF can be considered under submaximal - and so under quasi-isometric conditions (AFiso). Thereby AF is optimal, when the muscle produces exactly as much force as is applicated from the outside. In this case the forces cancel each other out. The static balance of forces remains.
- On the other hand, AF can be also considered under supramaximal (related to the maximal isometric force - and so under eccentric) - conditions, if the external load exceeds the maximal isometric Adaptive Force of the nerve-muscle system (supramaximal load; AFecc). At this, the drawback - through further force increase - shall occur as slowly as possible.

The concept of Adaptive Force is not only of academic importance. In case of injuries and so called overuse damages in sport and in working environment mostly the adaptive achievment to external forces plays a role. Almost all eccentric strain is associated with the development of muscle and tendon injuries. ${ }^{2-4}$ Biomechanical studies have shown the connection of deficits in eccentric force development and sport injuries - e.g. injuries of hamstrings in soccer or of ankle joint. ${ }^{5,6}$ Kieb et al. ${ }^{6}$ pointed out, that in animal experiments through concentric muscle action neither partial nor complete rupture of muscle fibres could be provoked, but through eccentric muscle action it could be caused. They charaterize the mechanism of injury as a "from the outside impacting force" which encounters "on a tensed in the length easing muscle (eccentric mechanism)". 3 These hints suggest that it is of particular importance for the integrity and damage resistance to react adequately to external impacting changeable forces. Thus, basic research on AF may lead to new insights.

\section{Materials and Methods}

\section{Measuring system for detection of Adaptive Force}

Needing a measuring system for the detection of Adaptive Force a valid pneumatic measuring method as well as a corresponding measuring apparatus were developed in cooperation with the company Seifert Drucklufttechnik GmbH Bernsbach Germany (Seifert pneumatic technique) (Fig. 1). The device is able to apply a defined varying force on the limb of the subject, who is trying to maintain a defined position against the machine as long as possible. The project was funded by the German Federal Ministry for Economic Affairs. In this paper the fundamental functioning and first reference measurements will be outlined. A full description of the measuring system and its quality will be published separately (typescript in preparation).

Besides the measurement of the maximal isometric force in the conventional sense $\left(\mathrm{FisO}_{\max }\right)$, the pneumatic measuring system allows the acquisition of the presented Adaptive Force (AF) with different force-time-courses. The basic principle is verification of how far the resistance of the subject against a varying force - generated from the system - differs from an ideal stable reference resistance (reference curve). Thereby, the AF is the better the closer the line 


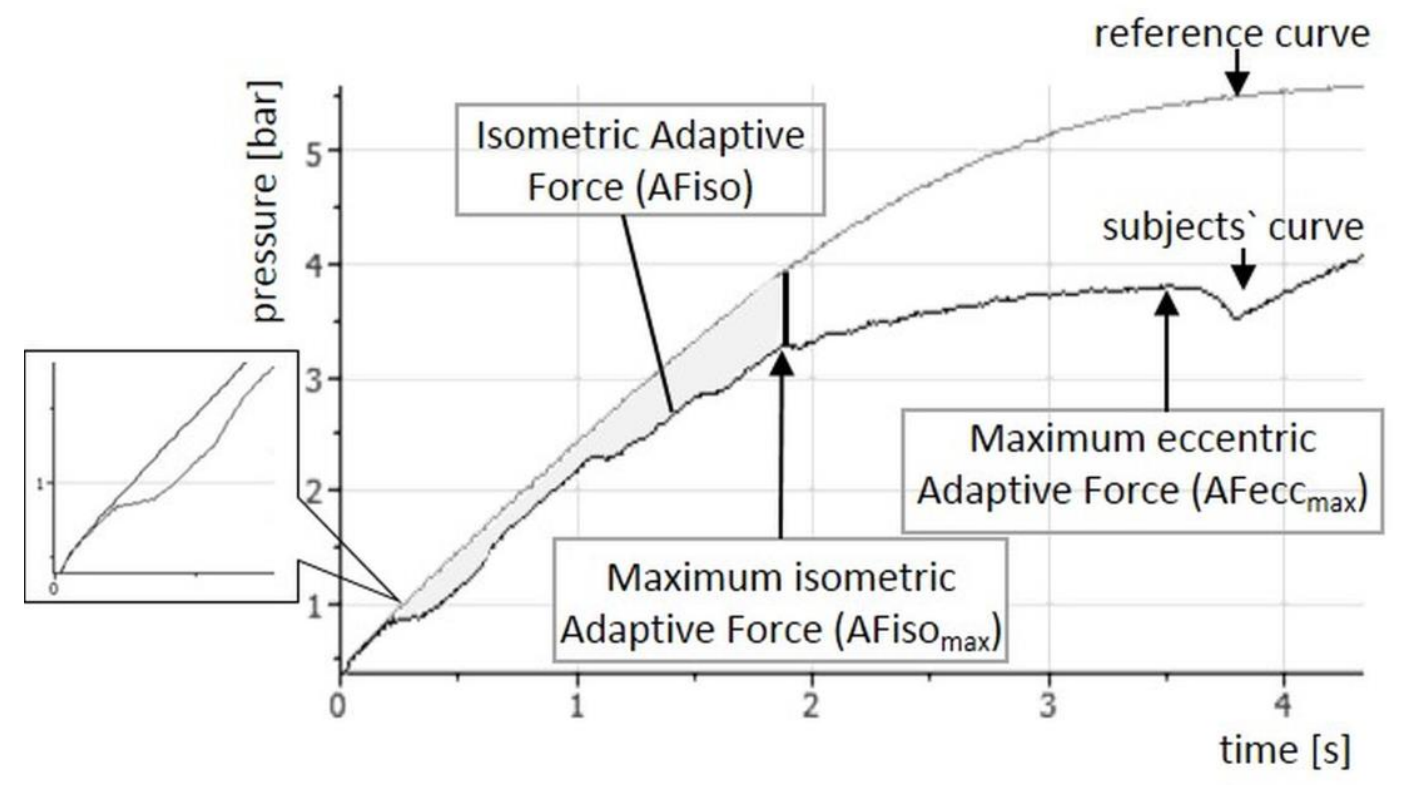

Fig 2. Course of pressure of an example measurement in relation to a reference curve [bar]; measured with SeBit knee ". The marked area is related to the AFiso.

of the pressures` graph produced by the subject runs along the course of the reference curve (Fig. 2).

The following distinctive stages and points with specific force qualities can be derived from the course of the measurement curve of the subject. The authors rank these items among the concept of AF.

\section{Initial response and isometric phase of AF (AFiso)}

After the start of the measurement the pressure increases in the measuring system. The pressure curve rises due to the muscular tension of the subject against the interface at the distal tibia. Thereby often a slight yielding of the leg occurs (in Fig. 2 at approximately $0.2 \mathrm{~s}$ ). If the AF has good quality, it then comes to static conditions: the increase in pressure in the cylinder is compensated by the subject. Here, the muscle works isometrically as long as the force of the quadriceps femoris muscle precisely matches to the pressure rise.

\section{Maximum isometric Adaptive Force (AFiso max $_{\text {) }}$ )}

The pure isometric phase is followed by shorter isometric phases, which are interrupted by intermittent rapid yielding motions. This iterative behavior in the transition from isometric to eccentric phase is largely due to technical reasons, since for slow movements friction and sliding phases quickly alternate. This occurs because of the piston seal of the measuring cylinder (slip-stick-effect). Strictly speaking, this already is a slow yielding movement. It is therefore necessary to specify, how long an isometric phase may last at least to still speak of isometry. Therefore, we defined an isometric phase for a period not less than
$0.25 \mathrm{~s}$. Shorter isometric phases are added to eccentric behavior. This is an arbitrary definition, which seems to correspond to the real transition given by the typical curve shape. The subject reaches the maximum isometric force $\left(\mathrm{AFiso}_{\max }\right)$ at the highest point of the isometric phase (as defined above) prior to the transition in eccentric contraction. This value

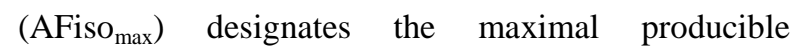
isometric force of the subject. At the same time it marks the transition to eccentric behavior (Fig. 2).

\section{Maximum eccentric Adaptive Force (AFecc max $_{\text {( }}$ )}

With exceeding the $\mathrm{AFiso}_{\max }$ the described drawback of the lower leg in direction of knee flexion follows. During the process of further increase in pressure, the friction phases are becoming scarcer until a continuous sliding motion of the push rod begins. That implies an eccentric action of the subjects' quadriceps femoris muscle. During this motion the largest muscular forces are generated, so that a further increase in pressure appears. Finally, this results in the maximum eccentric Adaptive Force (AFecc $\mathrm{max}_{\max }$ ). (Fig. 2)

Therefore, in one test sequence both the isometric and the eccentric form of the Adaptive Force can be quantified. Furthermore, the measurement may be performed with different slew rates of pressure. Since this has an impact on the results, the slew rates have to be standardized for the measurement procedure.

\section{Measurement procedure and ethics}

Since this manuscript basically includes the concept of the novel force form Adaptive Force, the measuring method and procedure is not described in detail. The 


\section{Adaptive Force, a specific neuromuscular function}

Eur J Transl Myol - Basic Appl Myol 2015; 25 (3): 183-189

Table 1. Illustration of gender and anthropometric data as well as first reference values of torque generated by the $\mathrm{AFecc}_{\max }$

\begin{tabular}{|c|c|c|c|c|c|c|c|}
\hline & $n$ & $\begin{array}{c}\text { age } \\
\text { [years] }\end{array}$ & $\begin{array}{l}\text { body height } \\
\text { [cm] }\end{array}$ & bodymass [kg] & $\begin{array}{c}\text { Torque } \\
{[\mathrm{Nm}]}\end{array}$ & $\begin{array}{c}\text { Torque } \\
{[\mathrm{Nm}]}\end{array}$ & $\begin{array}{c}\text { Torque per kg } \\
\text { bodymass } \\
{[\mathrm{Nm} / \mathrm{kg}]}\end{array}$ \\
\hline women & 17 & $\begin{array}{l}31.24 \\
\pm 9.97\end{array}$ & $\begin{array}{l}168.82 \\
\pm 5.77\end{array}$ & $\begin{array}{r}61.00 \\
\pm 6.32\end{array}$ & $\begin{array}{l}127.97 \\
\pm 35.06\end{array}$ & $\begin{array}{r}130.49 \\
\pm 34.18\end{array}$ & $\begin{array}{c}2.12 \\
\pm 0.52\end{array}$ \\
\hline men & 48 & $\begin{array}{c}25.4 \\
\pm 9.23\end{array}$ & $\begin{array}{l}181.14 \\
\pm 27.3\end{array}$ & $\begin{array}{c}80.38 \\
\pm 10.30\end{array}$ & $\begin{array}{r}205.65 \\
\pm 50.90\end{array}$ & $\begin{array}{r}206.86 \\
\pm 54.51\end{array}$ & $\begin{array}{c}2.57 \\
\pm 0.58\end{array}$ \\
\hline
\end{tabular}

arithmetic mean and standard deviation.

measuring system including quality criterion, measurement method and procedure, detailed information about the participants as well as a more extensively description of the results will be presented in detail in another article (typescript in preparation).

Since the measurements do not include any risks, impairments or intervention, there was no necessity of ethics committee according to the rules of procedure of the ethics committee of the University of Potsdam from the 20th October 2010. The test person provided their verbal consent to participate in this study after introducing them to the measurement system and procedure. The seven adolescent test persons were part of one training squad and were measured within a session in the presence of their coach, who had verbal consent by the parents.

\section{Results}

\section{Results of preliminary reference values}

The System SeBit knee was examined concerning the quality criteria. For describing the interrater-reliability, three different raters were compared measuring $n=3$ test persons by using the Intraclass Correlation Coefficient (ICC). Prior to this parametrical statistical test, the normal distribution of the data was checked utilizing the Kolmogorov-Smirnov-Test. The statistical analysis showed that the system fulfils the interraterreliability $(\operatorname{ICC}(3,1)=.974 ; \mathrm{p}=.00<\alpha=.05$; Cronbachs alpha $\alpha=.987)$. Furthermore, the test-retest reliability was examined over five weeks at $n=4$ test subjects measured bilaterally and analyzed with the ANOVA including a Greenhouse Geisser correction $(\mathrm{F}(4,20)=3.099, \mathrm{p}=.109, \alpha=.05)$. The validity was confirmed by a parallel test, which compared the used pressure sensor to a strain gauge (gold standard).

Since the data were not distributed normally, the Correlation Coefficient of Spearman was used and showed with $\rho=.976(p=.00)$ a very high correlation between both measurement methods. Until now a total of $n=65$ healthy subjects were measured for first reference values using the new measuring system. The sample consists of $n=17$ women and $n=48$ men. The eligibility criteria were defined in advance and according to the criteria the subjects were selected. The test subjects were aged between 15 and 54 years. They had no artificial joints and were free of complaints of knee joint since at least three month. Everyone completed a questionnaire containing personal and medical information. Table 1 shows the anthropometric data and the measured values of torque generated by the $\mathrm{AFecc}_{\max }$. The measuring system was adjusted so that the maximal measurement pressure of 6 bar was achieved within $5 \mathrm{~s}$. The maximum of the measurement curve of the subject is the $\mathrm{AFecc}_{\max }$. The torque was calculated by the determined force value and the measured lever length of the lower leg (distance between rotation axis in the center of the lateral condyle of femur and the middle of the contact of the interface at the tibia). As expected, the achieved absolute torque $(\tau)$ generated by the AFeccmax of women is lower than the values of men. The averaged relative torques of the knee extensor of female subjects $(2.12 \mathrm{Nm} / \mathrm{kg})$ and of male subjects $(2.57 \mathrm{Nm} / \mathrm{kg})$ show that the women generate averagely $82.49 \%$ of torque of men. Since in this article the focus lies on the concept of the Adaptive Force, the following discussion does not refer to the here briefly presented 


\section{Adaptive Force, a specific neuromuscular function}

Eur J Transl Myol - Basic Appl Myol 2015; 25 (3): 183-189

reference values, but to the idea of the introduced force concept of AF. A detailed description of the measuring system and the approval of its quality criteria will be given in a subsequent article (typescript in preparation).

\section{Discussion}

The authors postulate that the proposed concept of Adaptive Force (AF) is innovative. We didn't find yet a suitable description of the adaptability of the sensorimotor system in kinesiology. It seems to be a stand-alone concept of muscle action, which includes the adaptive behavior of isometric action under submaximal and maximal force conditions as well as the eccentric behavior under supramaximal conditions. The model of stretch-shortening cycle (SSC) cannot adequately reflect the Adaptive Force, because it includes the change from an eccentric to a concentric action. ${ }^{7}$ That means that - in contrast to the concept of $\mathrm{AF}$ - the SSC always is based on a counter-movement (eccentric to concentric). Furthermore, it solely takes place during rapid movements. This is even the case at the slow SSC. Moreover, the SCC does not consider the adaptive behavior during isometry and eccentric phase. Whereas the AF includes rapid as well as slow force progressions and involves pure isometry plus the transition between isometry and eccentric action.

\section{The isometric Adaptive Force (AFiso)}

What is new here is that the subject has to resist the force isometrically, which is generated through the measuring system and is variable. Thereby, the system allows movements. Whether movement will occur depends on the subject. The condition for this performance is on the one hand the force of the muscles involved. On the other hand, the setting requires a precise processing of proprioceptive inputs and the realization of an adequate force output, thus, a certain coordinative performance. During the submaximal isometric phase the human system is not at the limit of its force performance. In spite of rising force, the maintenance of the isometric status depends largely on the sensorimotor functionality. However, it is difficult to label the AF to a classic coordinative ability. The closest seems to be the kinesthetic differentiation ability according to Hirtz. ${ }^{8}$ But this includes also the accuracy of the movement performance during targeted actions and delicate motion sequences such as targeting throwing, swinging on the parallel bars. ${ }^{9}$ For a well pronounced kinesthetic differentiation ability it is crucial, how precisely the separated motion sections are coordinated, so that a harmonic movement may result. ${ }^{9}$ But the isometric Adaptive Force is just characterized by the absence of macro-movements. AFiso means that isometric conditions sustained for as long as possible, even though the extraneous force increases. Intersection with the kinesthetic differentiation ability is the continuous force coordination of the sensorimotor system matching with the external varying force during the measurement.

\section{The maximum eccentric Adaptive Force (AFecc $c_{\max }$ )}

The specialty of our approach is that the eccentric force is performed out of the isometry. It is determined, to which external force the nerve muscle system is able to suit to, even though it already yields. It has to be considered, that this yielding involves two dimensions of the muscular regulation: the regulation of force and of muscle length. Possibly, the sensorimotor quality depends on how good both - force and length regulation - work together. This hypothesis still has to be proved.

Since here the neuromuscular system has to adapt itself during the eccentric phase of $\mathrm{AF}$ (AFecc) to the constantly increasing force, there are coordinative amounts attributed to the AFecc, too.

Previous methods to measure the maximal eccentric force use high overload far above the resilience of the subject. The test person is forced by a desmodromic process of the measurement system and tries to oppose as much as possible. Accordingly, the force of an isokinetic system in the eccentric mode (maximum force) lies over the capabilities of the test person and the motion speed is fixed. ${ }^{10-12}$ It enforces a specific motion sequence to the neuromuscular system and does not allow an isometric increase in force (desmodromic!). Solely the adjustment of a threshold allows isometric action - but this still keeps desmodromic. An advantage of such a system is that the applied resistance of the subject is recorded over a defined angular range. Moreover, it can be measured with different angular velocities. However, the movements are forced. In addition, either eccentric or concentric or isometric measurements are possible. ${ }^{13}$ In contrast, during AF measurements a combination of isometric and eccentric action exists but a concentric measurement also is possible. Furthermore, the speed of motion in the concept of AF is a result of the force relation between man and machine and insofar variable. In this context, the AF-system seems to be closer to real-life processes. Regarding this point, the isokinetic and plyometric activities are clearly differentiated from the AF. It requires a separate definition for this specific force shape with focus on the adaptability of the neuromuscular system.

Another method for acquisition of eccentric forces was presented by Schmidtbleicher. ${ }^{14}$ Thereby the test person has to counteract a falling overload, which entails that the eccentric maximum is reached explosively. In contrast to this, the eccentric maximum of the system SeBit is achieved successively: the eccentric phase starts directly when exceeding the isometric maximum. Then it approaches ascending to the eccentric maximum until finally it exceeds this force. Thereby it ranges in the area of the eccentric 


\section{Adaptive Force, a specific neuromuscular function}

Eur J Transl Myol - Basic Appl Myol 2015; 25 (3): 183-189

maximum and does not work with an extreme overload far beyond this force value. It should be noted, that the test person is not able to retrieve the maximum force value of the measurement (during eccentric phase) at the end of the isometric phase, although it achieves those force values in the subsequent process of the measurement. At present it cannot be said, if this phenomenon is rather due to mechanical properties of the muscle or rather to the neurophysiological regulation. The authors opinion is that the AF owns a coordinative element, although it is strength ability. This agrees with the view of Farfel, ${ }^{15}$ who already classified the control of muscle strength to coordinative abilities in 1977. ${ }^{9}$ In the view of the authors, AF is to be understood as an integrative performance of the nerve muscle system: On one hand, it is based on conditional factors (submaximal, maximal and supramaximal strength). On the other hand, it requires the ability of kinesthetic differentiation, thus a coordinative component in conformity with Hirtz. ${ }^{8}$

\section{Neurophysiological View}

From the point of view of neurophysiology, generally all structures that are involved in motion control are participating during measurement of Adaptive Force (AF). Since the AF includes arbitrary processes, not only spinal structures are participated (short latency reflex), but also supraspinal ones (e.g. cortex, cerebellum, thalamus, basal ganglia).

Several authors suggested that eccentric muscle action involves unique control strategies of the central nervous system. ${ }^{16,17}$ This specific neuronal control is probably also active during Adaptive Force due to the following aspects: 1) AF includes an eccentric action. 2) The isometric phase of $\mathrm{AF}$ is performed in the holding mode.

In a dissertation ${ }^{18}$ it could be shown, that there are differences in the force endurance comparing a holding versus pushing mode of isometry. It is suggested, that the holding mode of isometry potentially has the same neuronal strategies as eccentric action, since functionally the holding mode seems to be closer to eccentric activity: in both muscle actions the aim is to resist an external force. It could be possible, that a particularly good adaptation to the increasing external force during the AF displays the capability of the specific control strategies during eccentric action. Duchateau \& Baudry state, that the unique mechanisms behind eccentric muscle action are unknown until today. ${ }^{19}$ The specific control strategies could be one reason for the benefits of eccentric training, e.g. ${ }^{20}$. Since Adaptive Force probably contains similar mechanisms, it could be useful for exercise, too, but possibly also concerning prevention, neuromuscular diagnostics or the like.

In conclusion, the term Adaptive Force (AF) aims to describe an aspect of neuromuscular functions that according to the authors - has not been considered sufficiently so far. Adaptive Force cannot be clearly classified into an already existing concept of kinesiology. It connects conditional and coordinative components of neuromuscular function. In the range of submaximal isometric action the coordination dominates, above this range, possibly, condition is predominant. The concept of AF distinguishes itself by a certain proximity to everyday processes. The most significant characteristic of the AF-concept, however, is that it describes the transition from isometric to eccentric contraction phases!

It can be assumed, that this approach has potential for practical applications. We will show that the measurement of AF by means of a pneumatic apparatus is simple, uncomplicated and inexpensive. Moreover, it offers a measurement quality sufficient for use in research, diagnostics, exercise training and therapy. Therefore, it is able to complement the established inventory of methods for force measurement. Especially, because it combines different forms of force during one measurement:

- Submaximal and maximal isometric Adaptive Force: AFiso/AFiso ${ }_{\text {max }}$

- Eccentric Adaptive Force (slow/rapid): $\mathrm{AFecc}_{\max } /$ rapAFecc $_{\text {max }}$

Further, it allows also to measure the maximal isometric force in the traditional sense by pushing isometrically against a stable resistance: Fiso $_{\max }$.

In future works we will show that, during rapid force, increased supramaximal Adaptive Forces can be generated. ${ }^{21}$ This may be used for performanceoriented training, a promising application.

We will propose, that isometric action - in the strict sense of the term isometry as force production under really stable position - does not exist. We will show that at least two, possibly three, different forms of isometric actions can be performed and that each is measurable with the new pneumatic device here presented.

\section{Acknowledgements}

This project was funded by the Federal Ministry of Economy and Technology (Project ZIM KF2262301FO9).

\section{Authors contributions}

All authors participated in the theoretical concept development. $\mathrm{MH}$ and LS constructed the measuring system and did the evaluation of the system, measurements of participants and analysis of the data. $\mathrm{MH}, \mathrm{LS}$ and FB were involved in interpretation of the data and in writing the report. All authors revised the manuscript.

\section{Disclosures about potential conflict of interest}

The employment in 2011 of two authors was funded by the Federal Ministry of Economy and Technology. But there are no potential conflict of interest. 


\section{Adaptive Force, a specific neuromuscular function}

Eur J Transl Myol - Basic Appl Myol 2015; 25 (3): 183-189

\section{Corresponding Author}

Laura Schaefer

Karl-Liebknecht-Str. 24-25, Haus 24, R. 1.14

14476 Potsdam, Germany. Phone: +49-331-977 2987

E-mail: 1schaefe@uni-potsdam.de

E-mails of CoAuthors

Marko Hoff: marko.hoff@gmail.com

Nancy Heinke: heinke@uni-potsdam.de

Frank Bittmann: bittmann@uni-potsdam.de

\section{References}

1. Schnabel G, Harre D, Borde A. Trainingswissenschaft. Berlin: Sportverlag 1997.

2. Garrett WE Jr. Muscle strain injuries: clinical and basic aspects. Med Sci Sports Exerc 1990;22:43643.

3. Kieb M, Lorbach O, Engelhardt $\mathrm{M}$. Muskelverletzungen: Diagnostik und Behandlungen. Der Orthopäde 2010;39:1098107.

4. Murgia A, Harwin W, Prakoonwit S, Brownlow H. Preliminary observations on the presence of sustained tendon strain and eccentric contractions of the wrist extensors during a common manual task: implications for lateral epicondylitis. Med Eng Phys 2011;33:793-7.

5. Fousekis K, Tsepis E, Poulmedis P, et al. Intrinsic risk factors of non-contact quadriceps and hamstring strains in soccer: a prospective study of 100 professional players. $\mathrm{Br} \mathrm{J}$ Sports Med 2011;45:709-14.

6. Witchalls J, Blanch P, Waddington G, Adams R. Intrinsic functional deficits associated with increased risk of ankle injuries: a systematic review with meta-analysis. Br J Sports Med 2011. doi:10.1136/bjsports-2011-090137

7. Komi PV. Der Dehnungs-Verkürzungs-Zyklus. In: Komi PV, editor. Kraft und Schnellkraft im Sport. Köln. Deutscher Ärzte Verlag 1994;17382.

8. Hirtz P. Koordinative Fähigkeiten im Schulsport Berlin: Volk und Wissen, 1988.

9. Bertram AM, Laube W. Sensomotorische Koordination. Stuttgart: Thieme Verlag, 2008.

10. Weineck J. Optimales Training. Leistungsphysiologische Trainingslehre unter besonderer Berücksichtigung des Kinder- und Jugendtrainings. 14th ed. Balingen: Spitta Verlag, 2004.
11. Kannus P. Isokinetic Evaluation of Muscular Performance: Implications for Muscle Testing and Rehabilitation. Int $\mathbf{J}$ Sports Med 1994;15:S11-18.

12. Anderson MA, Gieck JH, Perrin D et al. The Relationships among Isometric, Isotonic, and Isokinetic Concentric and Eccentric Quadriceps and Hamstring Force and Three Components of Athletic Performance. Journal of Orthopaedic Sports Physical Therapy 1991;14:114-20.

13. Tredinnick TJ, Duncan PW. Reliability of Measurements of Concentric and Eccentric Isokinetic Loading, Physical Therapy. 1988;68:656-59.

14. Bührle M, Schmidtbleicher D, Ressel H. Die spezielle Diagnose der einzelnen Kraftkomponenten im Hochleistungssport. Leistungssport. 1983; 3: 11-6.

15. Farfel VS. Bewegungssteuerung im Sport. Berlin: Sportverlag, 1977

16. Enoka RM. Eccentric contractions require unique activation strategies by the nervous system. J Appl Physiol 1996;81:2339-46.

17. Grabiner MD, Owings TM, George MR, Enoka RM . Eccentric contractions are specified a priori by the CNS. Proc VXth Congr Int Soc Biomech Jyva“skyla“ Finland July 2-6, 1995, 338-9.

18. Schaefer L. Synchronisationphänomene myotendinöser Oszillationen interagierender neuromuskulärer Systeme - mit Betrachtung einer Hypothese bezüglich unterschiedlicher Qualitäten isometrischer Muskelaktion. Dissertation. Publikationsserver Universität Potsdam, 2014. http://opus.kobv.de/ubp/volltexte/2014/7244/

19. Duchateau J, Baudry S. Insights to the neural control of eccentric contractions. J Appl Physiol 2014;116:1418-25. DOI: 10.1152/japplphysiol. 00002.2013

20. Grabiner MD, Owings TM. Effects of eccentrically and concentrically induced unilateral fatigue on the involved and uninvolved limbs. Journal of Electromyography and Kinesiology 1998;9:185-9.

21. Schaefer L, Hoff M, Barnick D, Bittmann F. Einfluss der muskulären Vorspannung auf die maximale schnelle Adaptive Kraft: Der BoostEffekt. Deutsche Zeitschrift für Sportmedizin 2012;63:200. 